\title{
Die akute und chronische Außenbandinstabilität am Sprunggelenk
}

\author{
Dara Orangi, Karl Heinrich Winker
}

\section{Zusammenfassung}

Die akute Außenbandinstabilität ist meist die Folge eines Supinationstraumas. Heute wird die akute Außenbandinstabilität bis auf wenige Ausnahmen konservativ (früh funktionell) behandelt. Die chronische Instabilität ist die Folge einer meist konservativ vorbehandelten oder unbehandelten Kapsel-BandVerletzung des oberen Sprunggelenkes. In der Behandlung der chronischen Instabilität muss zwischen der mechanischen und der funktionellen Instabilität unterschieden werden. Die mechanische Instabilität wird operativ, die funktionelle konservativ behandelt.

\section{Einleitung}

Das Körpergewicht wird über die beiden oberen Sprunggelenke auf die Sprungbeine geleitet, das Stehen und Gehen ist ein dynamischer Vorgang, der Wechsel zwischen Abrollbewegung und Ruhen des Fußes auf dem ebenen oder unebenen Boden setzt einen anpassungsfähigen stabilen Kraftträger voraus. Bei der Insuffizienz bereits einzelner Zügel des lateralen Seitenbandapparates des oberen Sprunggelenkes sind deutliche Störungen des Gangbildes nachweisbar. In Anbetracht der Inzidenz des Umknicktraumas und der sozioökonomischen Relevanz bleibt die Instabilität des Sprunggelenkes als Diskussionsthema immer aktuell.

OP-JOURNAL 2001; 17: 24-30

(C) Georg Thieme Verlag Stuttgart · New York
Die Insuffizienz bereits einzelner Zügel des lateralen Kollateralbandapparates des oberen Sprunggelenkes führt zur deutlichen Störung des Gangbildes.

\section{Anatomie und Kinematik des Kapsel-/ Bandapparates}

Stabilisatoren des oberen Sprunggelenkes

- Der wichtigste Stabilisator des als Scharniergelenk angelegten oberen Sprunggelenkes ist sein knöcherner Aufbau. Die distalen Enden von Tibia und Fibula, die gemeinsam eine $\mathrm{Ge}$ lenkpfanne in Form einer Hohlrolle bilden, sitzen auf der gekrümmten konvexen Sprungbeinrolle auf. Die Achse des oberen Sprunggelenkes verläuft vom Innenknöchel zum Außenknöchel, dadurch ist die Dorsal- und Plantarflexion des Fußes gegen den fixierten Unterschenkel möglich.

- Als 2.Stabilisator dient der KapselBand-Apparat. Die Kapsel des oberen Sprunggelenkes setzt an Fibula und Tibia an, umhüllt die Trochlea tali, ist an der Vorder- und Hinterwand dünn, an den seitlichen Wänden durch den medialen und lateralen Bandkomplex verstärkt. Der laterale Bandapparat besteht aus drei Bändern:

1. Das ligamentum fibulotalare anterius als schwächstes Glied des Komplexes.
Das Band zieht von der Außenknöchelspitze zur seitlichen Fläche des Talus. Es sichert das Gelenk gegen laterale Aufklappbarkeit und Verschiebung des Sprungbeines nach vorne.

2. In der Mitte zieht das Ligamentum fibulocalcaneare. Von der Außenknöchelspitze zur äußeren Fläche des Fersenbeines ziehend, stabilisiert dieses Band gegen laterale Aufklappbarkeit und entsprechend seines Verlaufes das hintere untere Sprunggelenk.

3. Das Ligamentum fibulotalare posterius verbindet die Hinterkante des Sprungbeines mit der Außenknöchelspitze und verhindert somit eine Verschiebung des Talus nach hinten (Abb.1).

- Der dritte Stabilisator ist die reflektorisch kontrollierte, aktive muskuläre Reaktion. Eine Überdehnung des Kapsel-Band-Apparates führt zur reflekorischen Kontraktur der Muskulatur. Im Falle eines lateralen Aufklappens werden z.B. als entgegenwirkende Schutzmaßnahme die Pronatoren aktiviert.

Der mediale Bandapparat besteht aus dem Ligamentum Deltoideum (Deltaband). Dieses Band teilt sich in eine oberflächliche und in eine tiefe Schicht. Die oberflächliche Schicht besteht aus 3 Teilen:

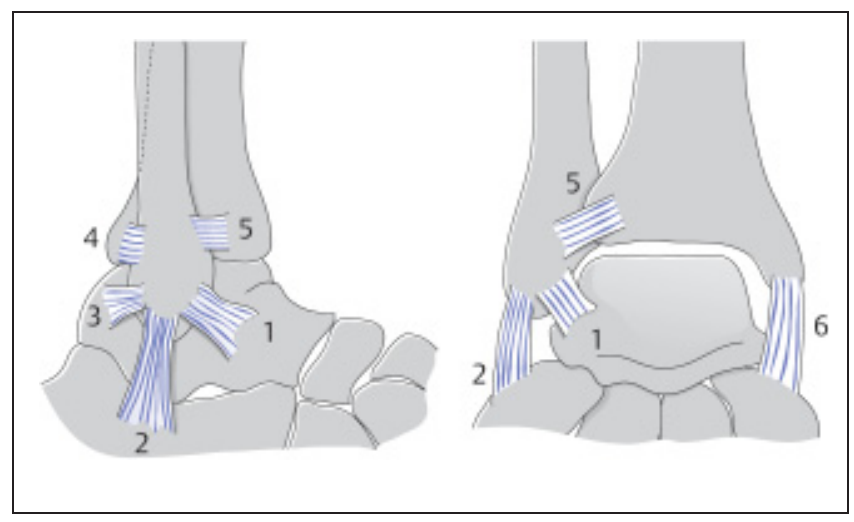

Abb.1 1: Ligamentum fibulotalare anterius, 2: Ligamentum fibulocalcaneare, 3: Ligamentum fibulotalare posterius, 4: hintere Syndesmose, 5: vordere Syndesmose, 6: Ligamentum deltoideum. 
1. Verbindung zwischen Innenknöchel und Os naviculare: Pars tibionavicularis.

2. Ligamentäre Verbindung zwischen Fersenbein und Innenknöchel: Pars tibiocalcaneare.

3. Ligamentäre Verbindung zwischen Innenknöchel und Sprungbein: Pars tibiotalare posterius.

Die tiefe Schicht verbindet Tibia und Talus. Die pars tibiotalare anterius verläuft unter dem Ligamentum tibionaviculare [1].

Stabilisatoren des oberen Sprunggelenkes:

das Skelett

- der Kapsel-Band-Apparat

- der Reflexbogen

\section{Kinematik}

Die Bänder des oberen Sprunggelenkes sichern die statische und dynamische Stabilität des Ganges. Jede Bewegung in entgegengesetzter Richtung des Bandverlaufes wird in der Endlage gehemmt.

Bei Dorsalflexion wird einerseits aufgrund der Anatomie des Talus (die Sprungbeinrolle ist in ihrem vorderen Anteil 4-5 $\mathrm{mm}$ breiter), andererseits durch erhöhte Anspannung der Bänder eine „close-packed-position“ (gesperrte Stellung) erreicht. In Dorsalflexion kommt es neben der Bandanspannung durch die Außenrotation zu einer Torsionsspannung im distalen tibiofibularen Gelenk. Es wird ein federnder Widerstand erreicht, der insbesondere in der Abstoßphase benötigt wird.

Die Plantarflexion wird durch die Anspannung folgender Bänder dynamisch unterstützt:

Lig. tibiotalare anterius, Lig. tibionaviculare, Lig. fibulotalare anterius. Die zentrale Aufgabe des medialen Bandapparates ist die Stärkung der Verbindung zwischen Unterschenkel und Fuß und somit hauptsächlich statisch. Die antagonistische Wirkung der Muskulatur ist eine weitere stützende Säule der Dynamik des oberen Sprunggelenkes. In Dorsalflexion ist es insbesondere die angespannte Achillessehne und die kontrollierte Kontraktion des M. triceps surae. In Plantarflexion sind es die Dorsalextensoren, insbesondere der M. tibialis anterior und die Peroneusgruppe [2].

\section{Verletzungsmechanismus}

\section{Das Supinationstrauma}

Es handelt sich um einen kombinierten Verletzungsmechanismus, die Erstbeschreibung geht auf Kaufmann 1922 zurück (zit. in [3]). In den meisten Fällen berichten die Unfallverletzten vom Umknicken mit dem Fuß: meistens ist der innere Fußrand angehoben, der Fuß einwärts gedreht, das obere Sprunggelenk plantarflektiert (Supinations-Adduktions-Inversions-Trauma). Die Inzidenz in der Gesamtbevölkerung liegt bei $1 \mathrm{zu} 10000$, ist allerdings geschlechts- und altersabhängig [4]. Für junge männliche Sportler unter 40 Jahre errechnete Holmer in Dänemark eine Inzidenz von 7 auf 1000 [5]

Die meisten Distorsionsverletzungen des oberen Sprunggelenkes entstehen beim Sport (15\% aller Sportunfälle betreffen den Außenbandapparat des oberen Sprunggelenkes), an zweiter Stelle stehen Haushalts-und Freizeitunfälle, danach folgen die Arbeitsunfälle [4,5].

Entsprechend diesem Trauma-Mechanismus ist eine so genannte Supinationslinie beschrieben worden. Auf dieser Linie befinden sich die meisten Begleitverletzungen nach einem Umknicktrauma [6] (Abb. 2).

\section{Klinische Symptomatik}

\section{Akute Instabilität}

Schwellung des Sprunggelenkes sowie ein Druckschmerz vor allem im Verlauf des Lig. fibulotalare anterius sind die typischen klinischen Zeichen nach einem Supinationstrauma. Die klinische Prüfung darf nicht auf die Knöchel-Region beschränkt sein, alle charakteristischen Schmerzpunkte müssen aufgesucht und untersucht werden (Abb.3 u.4).

Begleitverletzungen:

Frakturen:

- Metatarsale V - Basisfraktur

- Talusläsionen

- Kalkaneusfraktur

- Fraktur os cuboideum

- os navikulare

- Innen- oder Außenknöchelfraktur, Epiphysiolyse dist. Fibula

- Bandverletzungen:

- Innenbandapparat

- peroneales Retinakulum

- vordere tibiofibulare Syndesmose

- Kalkaneocuboidales Band

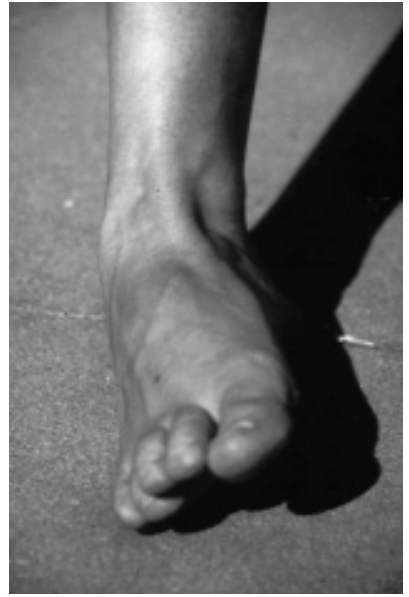

Abb. 2 Umknicktrauma: Supination-/Adduktion.
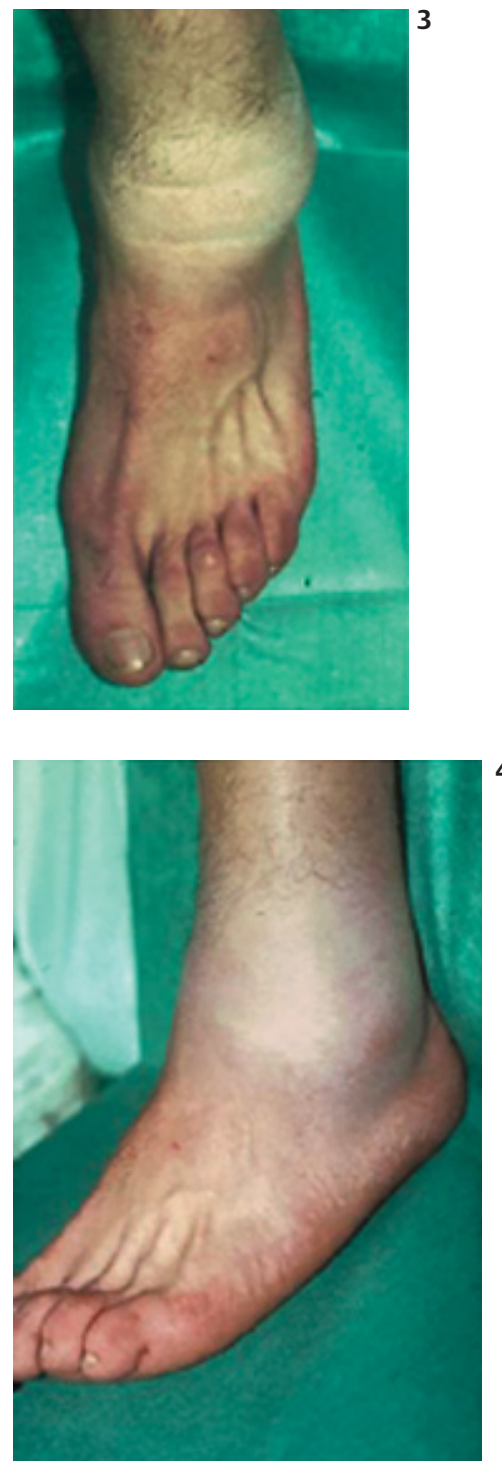

4

Abb. 3 u. 4 Schwellung über dem gesamten Sprunggelenk nach Umknicktrauma. 

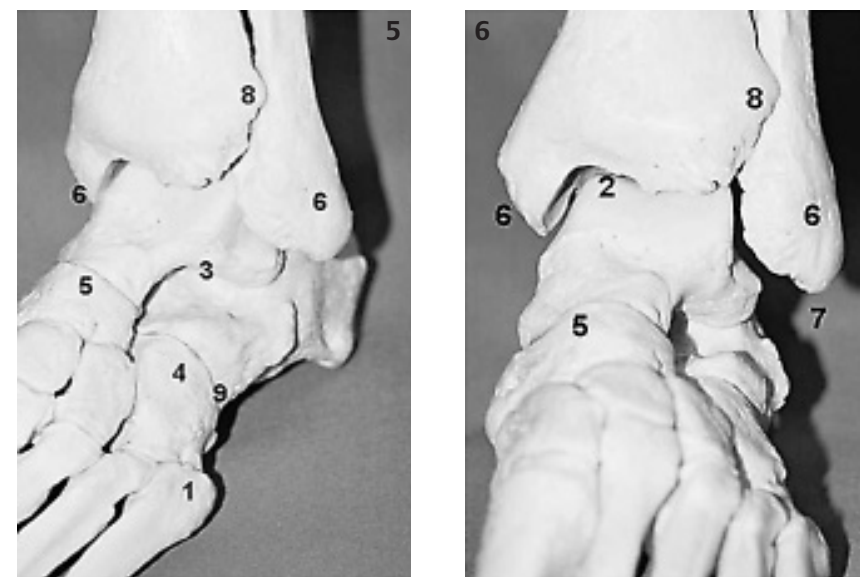

Abb. 5 u. 6 1: Basis MT V2: Talus 3: Calcaneus, 4: Os cuboideum, 5: Os naviculare, 6: Innenband/ Innen- und Außenknöchel, 7: Peroneales Retinakulum, 8: vordere Syndesmose, 9: calcaneocuboidales Band.

Die klinische Stabilitätsprüfung ist in der Akutphase schmerzhaft und sollte nach Abklingen der Akutsymptomatik erfolgen. Klinisch ist die Stabilitätsprüfung des OSG von größter Bedeutung. Für die Prüfung der vorderen Schublade wird die Ferse des verletzten Fußes bei fixiertem Unterschenkel nach vorne gedrückt, eine Verschiebung des Talus nach vorne ist bei Instabilität fast immer tastbar, manchmal auch sichtbar. Zur Prüfung der Aufklappbarkeit wird die Ferse nach medial gedrückt (Abb. 7 u.8).

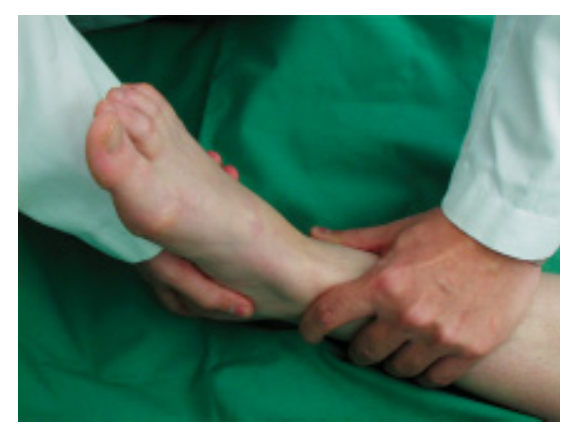

Abb.7 Klinische Stabilitätsprüfung: vordere Schublade.

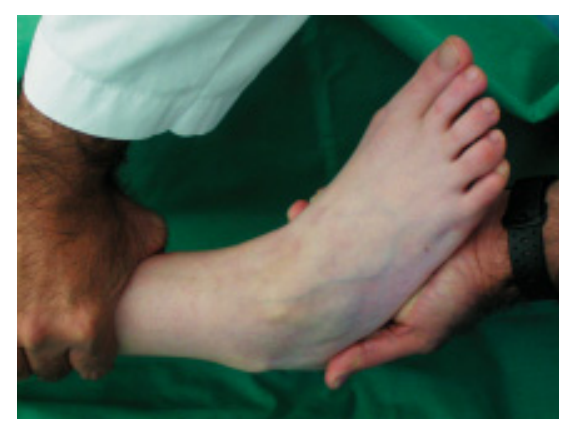

Abb.8 Klinische Stabilitätsprüfung: laterale Aufklappbarkeit.

\section{Diagnostik}

Akute Instabilität

Nach der klinischen Untersuchung werden Röntgen-Aufnahmen des oberen Sprunggelenkes in 2 Ebenen angefertigt. Bei Verdacht auf Begleitverletzungen (Abb.5 u.6) müssen radiologische Untersuchungen des verletzten Unterschenkels/Fußes folgen [7].

Die radiologische Stabilitätsdiagnostik wurde schon 1933 von Dehne als gehaltene Aufnahmen in 2 Ebenen für die Erkennung der traumatischen Instabilität angegeben (zit. in [3] ). Die Stressaufnahmen zur Bestimmung des Talusvorschubes und der lateralen Aufklappbarkeit können standardisiert mit definierter Inversionskraft von $15 \mathrm{kp}$ (gehaltene Aufnahmen nach Prof. Scheuba) durchgeführt werden [8]. Ihre Bedeutung wird heute nur noch eingeschränkt gesehen. Durch die schmerzbedingte reflektorische Anspannung vor allem der Peroneal-Muskulatur entstehen falsch negative Röntgenbefunde.

Indikation für die gehaltene Aufnahme - chronische Instabilität

- bei klinischem Verdacht auf eine akute Instabilität nach Abklingen der Akutsymptomatik

- Kontrolle nach konservativer Therapie

Die Grenzwerte der vorderen Schublade und des Talusvorschubes werden in der Literatur unterschiedlich angegeben. In unserer Klinik wird eine Aufklappbarkeit bis zu 5 Grad im Seitvergleich als normal bewertet, Werte zwischen 6 und $15 \mathrm{Grad}$ werden als Zeichen einer Einbandruptur gedeutet, eine Aufklappbarkeit über 15 Grad ist für uns Hinweis (nicht Beweis) auf das Vorliegen einer 2-Band-Ruptur.
Bei einer Aufklappbarkeit von 30 Grad und mehr (immer im Vergleich zur gesunden Seite) liegt vermutlich eine 3Band-Ruptur vor.

Ist die klinische Symptomatik nicht spezifisch, liegt der Verdacht auf eine okkulte Fraktur oder osteochondrale Läsion des Talus vor. Es sollte zur weiteren Abklärung ein MRT angefertigt werden [8].

Die Sonographie hat keinen Eingang in die klinische Routine gefunden, die Arthrographie gehört der Vergangenheit an.

Bei über 90\% der Kapsel-Band-Verletzungen des oberen Sprunggelenkes ist das Ligamentum fibulotalare anterius rupturiert, bei ca. $2 / 3$ der Fälle ist eine Ruptur des ligamentum fibulocalcaneare nachweisbar, das Lig. fibulotalare posterius ist in $10 \%$ der Fälle gerissen. Etwa $75 \%$ der Außenbandrupturen sind intraligamentär, knöcherne Bandausrisse kommen besonders häufig bei Kindern und Jugendlichen vor. Die Häufigkeit der einfachen und der kombinierten Außenbandverletzungen wurde von Zwipp bei 1235 Patienten untersucht [9] (Tab.1).

Isolierte Verletzungen des medialen Bandapparates, der distalen tibiofibularen Syndesmose mit ihrem vorderen, unteren oder hinteren Bandanteil sind selten und kommen ohne Frakturbeteiligung noch seltener vor [11] (Abb.9).

\section{Therapie der akuten anterolateralen Rotationsinstabilität des oberen Sprunggelenkes}

Das Ziel der Therapie ist die Wiederherstellung der Stabilität. Anfang des 20. Jahrhunderts wurde die Bandverlet-

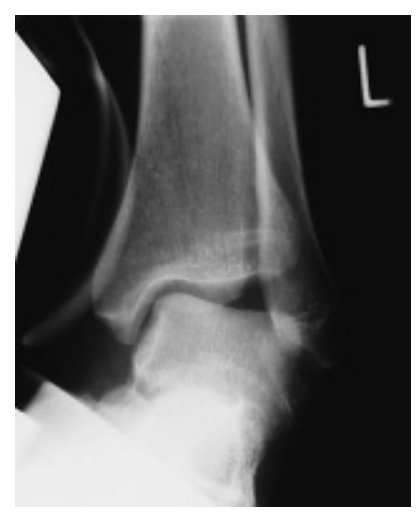

Abb.9 Laterale Aufklappbarkeit: Der Winkel der Gelenkflächen des Schien- und Sprungbeines: 16 Grad laterale Aufklappbarkeit. 
Tab. 1. Die Häufigkeit der einfachen und der kombinierten Außenbandverletzungen nach Prof. Zwipp

\begin{tabular}{lcc} 
Bandruptur & Anzahl (n) & Anzahl (\%) \\
\hline isoliert & & \\
Fibulotalare anterius (FTA) & 258 & 20,9 \\
Fibulocalcaneare (FC) & 5 & 0,4 \\
\hline kombiniert & & \\
FTA + FC & 910 & 73,7 \\
FTA + FTP & 38 & 3,1 \\
FTA + FC + FTP & 24 & 1,9
\end{tabular}

Aus [10]

zung konservativ behandelt, die operative Behandlung wurde nach deutscher Literatur 1927 von Katzenstein, nach dem englischen Schrifttum 1928 von Elmslie und nach der französischen Literatur 1934 von Seneque gefordert. Erst in den 80er Jahren wurden vergleichbar gute Resultate nach funktionell konservativer Behandlung veröffentlicht und stellten die primär operative Therapie infrage (zit. in [9]).

Heute wird die akute Außenbandinstabilität bis auf wenige Ausnahmen konservativ (früh funktionell) behandelt. Die Vorgeschichte des Patienten, die eingeschätzte Verletzungsschwere und die diagnostizierten Begleitverletzungen einerseits sowie Beruf, Alter und sportliche Ambitionen des Patienten andererseits sind diesbezüglich die Entscheidungshilfen. Bei entsprechender klinischer Symptomatik und auffälligem Röntgenbefund muss zum Ausschluss einer osteochondralen Läsion (meist an der medialen Talusrolle) eine MRTUntersuchung angeschlossen werden $[8,10]$.

Wir sind der Meinung, dass bei den genannten wenigen Ausnahmeindikationen die operative Therapie und anschließende funktionelle Nachbehandlung mit kurzer Immobilisationszeit von 4 Wochen die beste therapeutische Alternative für den Patienten darstellt.

Entscheidungshilfen zur operativen The-
rapie:
- 3-Band-Ruptur
- knöcherne Bandausrisse
- osteochondrale Talusläsion
- stattgehabte Bandverletzungen/
Operationen
- sportliche Ambitionen
- Patientenalter

Im Falle der Indikation zur konservativ frühfunktionellen Therapie wird zur Be- handlung des Verletzungsödems und Ruhigstellung der verletzten Bänder ein gespaltener US-Liegegips angelegt. Nach Abschwellen der Weichteile belastet der Pat. schmerzabhängig in einer Sprunggelenksorthese. Das Ziel sollte die frühestmögliche Vollbelastung des Beines sein. $\mathrm{Ab}$ dem Tage der Vollbelastung wird die vom ersten Immobilisationstag begonnene Thromboseprophylaxe beendet. Die stabilisierende Orthese wird für insgesamt 6 Wochen getragen.

Eine Metaanalyse aller Veröffentlichungen zwischen 1966 und 1998 betreffend der Behandlung der Außenbandruptur des oberen Sprunggelenkes in der JuniAusgabe 2000 des amerikanischen „The Journal of Bone and Joint Surgery" bestätigt die oben aufgeführte und in Deutschland allgemein akzeptierte Behandlungsstrategie [13].

\section{Op.-Technik}

Die Inzision verläuft gerade über der distalen Fibula und biegt über dem Außenbandapparat leicht nach vorne um. Der N. peroneus superficialis muss geschont werden. Auf dem Niveau des Periostes an der Außenknöchelspitze wird der Bandapparat mit seinen 3 Anteilen dargestellt und auf Stabilität geprüft. Meist liegt eine Ruptur der ventralen Kapselanteile vor. Hämarthros muss beseitigt, das Gelenk mehrfach ausgespült werden. Als erster Schritt wird die Stabilität des ligamentum fibulotalare posterius und der Syndesmose geprüft. Liegt ein knöcherner Bandausriss vor, erfolgt die stabile Refixation mit Kleinfragmentspongiosaschraube. Alternativ kann die transossäre Naht oder Knochenankernaht (z. B. Mit$\mathrm{ek}^{\circledR}$ ) die anatomische Situation wiederherstellen. Erfahrungsgemäß liegt in den meisten Fällen eine intraligamentäre Ruptur des Ligamentum fibulotalare anterius und des fibulocalcanearen Bandes vor, welche mit U-Nähten mit resorbier- barem Nahtmaterial (z.B. PDS 2/0) versorgt werden.

Postoperativ erfolgt die Ruhigstellung im US-Liegegips mit Wund- und Bewegungsfenster ab dem 2. postoperativen, Fadenzug am 11. p.o. Tag. Fortführung der Ruhigstellung für insgesamt 4 Wochen postoperativ im US-Gehgips mit Absatzerhöhung der Gegenseite. Nach Gipsabnahme wird ein Unterschenkelgummistrumpf der Kompressionsklasse I für weitere 4 Wochen verordnet. Währenddessen ist bei Sportlern medizinische Trainingstherapie möglich. Danach kann Leistungssport wieder betrieben werden.

\section{Chronische Instabilität}

Die chronische Instabilität des lateralen Sprunggelenkes als Spätfolge nach fibularen Kapsel-Band-Läsionen ist durch eine subjektiv und objektiv bestehende Instabilität mit Umknickneigung, Schwellungszuständen und Belastungsschmerzen gekennzeichnet [14].

Die mechanische Instabilität aufgrund unterbrochener ligamentärer Verbindung zwischen Fibula, Talus und Kalkaneus muss von der funktionellen Instabilität (fehlende Fähigkeit der muskulären und reflektorischen Reaktion auf ein Umknicktrauma) unterschieden werden $[15,16]$. Diese Unterscheidung ist von großer Bedeutung, weil sie über operatives oder konservatives Vorgehen entscheidet.

Die Anamnese liefert wichtige Informationen über die Form und Dauer der Therapie nach stattgehabter Kapsel-BandVerletzung, die Häufigkeit des Umknickens, die möglichen beruflichen und sportlichen Aktivitäten, das Ausmaß der Beweglichkeit, das Gangbild. Das Ergebnis der klinischen Stabilitätsprüfung, Schwellungszustand, Überwärmung und Druckschmerzhaftigkeit müssen dokumentiert werden. Eine exakte Rö.-Untersuchung des oberen Sprunggelenkes in 2 E. (Arthrose?, freier Gelenkkörper?, knöcherne Bandausrisse?), standardisierte gehaltene Aufnahmen des oberen und evtl. des subtalaren Gelenkes (bei v.a. Instabilität des hinteren unteren Sprunggelenkes) und evtl. MRT-Untersuchung bei V.a. Begleitverletzungen und bei vorangegangener Bandplastik müssen angefertigt werden.

Die nachfolgende Tabelle stellt die Wertigkeit der subjektiven und objektiven Zeichen in der Unterscheidung zwischen 
Tab. 2. Die Wertigkeit der subjektiven und der objektiven Zeichen der mechanischen und der funktionellen Instabilität

\begin{tabular}{lll} 
& mechanische Instabilität & funktionelle Instabilität \\
\hline Hypermobilität & eher ja & eher nein \\
eingeschränkte Beweglichkeit & eher nein & eher ja \\
Schwellung & eher ja & eher nein \\
Belastungsschmerz & ++ & + \\
Giving way-Symptomatik & + & ++
\end{tabular}

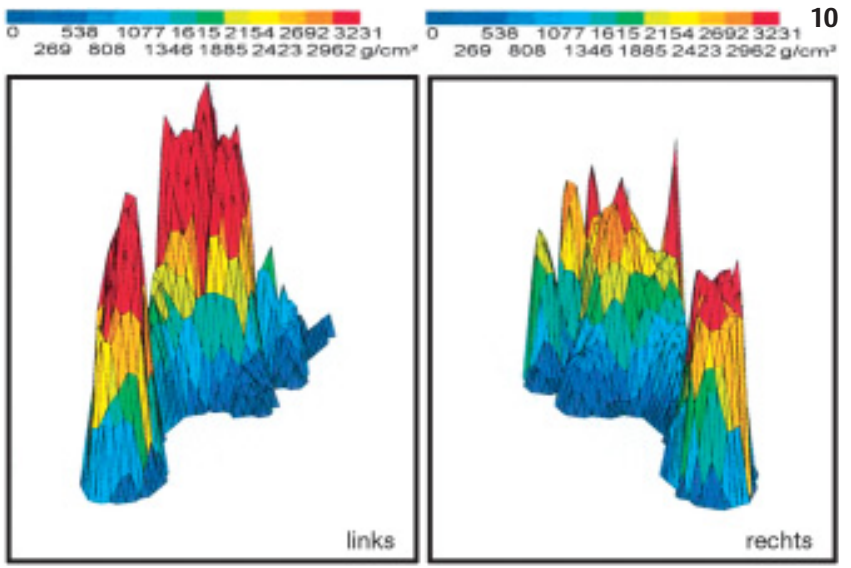

Abb.10 dynamische Pedographie: erhöhte Druckbelastung des lateralen Fußrandes links bei funktioneller Instabilität.

funktioneller und mechanischer Instabilität dar. Es handelt sich dabei um in der Literatur angegebene, statistisch nicht verwertbare Angaben (Tab. 2).

Die Reaktionsfähigkeit der Pronatoren als dynamische Stabilisatoren des OSG kann mit Hilfe moderner computergestützter Messverfahren objektiviert werden. Zu den neuen diagnostischen Verfahren, die sich alle aufgrund der zeitintensiven Messungen und des hohen Anschaffungspreises nicht durchsetzen konnten, gehören: die dynamische Pedographie, die Kippplattformmessung der peronealen Reaktionszeit sowie die Stabilometrie und Kraftmessung der Pronatoren.

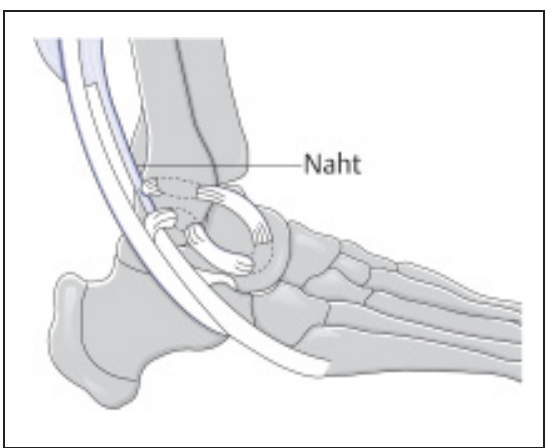

Abb.11 Schematische Darstellung der Watson-Jones-Plastik, Modifikation nach Holz-Weller.
Die Unterscheidung zwischen mechanischer und funktioneller Instabilität entscheidet über operatives oder konservatives Vorgehen.

In unserer Klinik werden die dynamische Pedographie und Ganganalyse eingesetzt. Die medial betonte, verlagerte mittlere Druckverteilung ist als Zeichen der mechanischen und die Lateralverlagerung der Druckverteilung als Zeichen der funktionellen Instabilität zu werten [17]. Die erhöhte Außenrand-Belastung des linken Fußes zeigt die Abb.10.

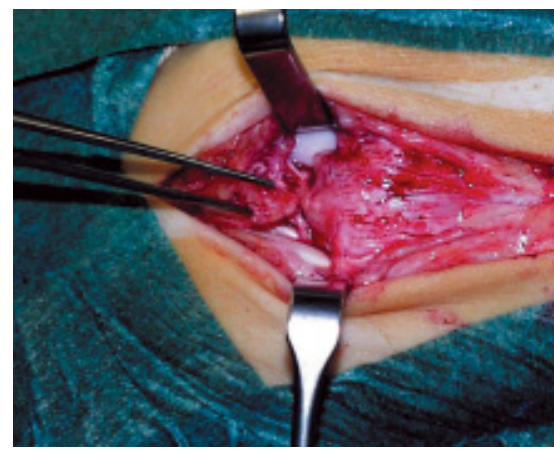

Abb.12 Verkalkung im Verlauf des rupturierten lig. fibulocalcaneare.

\section{Operative Therapie}

Ist die mechanische Instabilität nachgewiesen, hat ein auf den Patienten abgestimmtes Schulungsprogramm: Gangschule, Koordinationstraining und Reflextraining (Pronatoren betont) keine Besserung erbracht, ist die operative Therapie mit bandplastischem Verfahren die Methode der Wahl. Werden nahtfähige Stümpfe vorgefunden, sollte die anatomiegerechte Wiederherstellung der Bandkontinuität erfolgen. Die Periostlappenplastik stellt eine weitere Behandlungsalternative dar.

Unter den Tenodese-Verfahren zeigt die Watson- Jones-Plastik einen nahezu anatomischen Transplantatverlauf, das ligamentum fibulotalare anterius wird ersetzt. Tenodesen mit einem extraanatomischen Verlauf führen zur Störung des Wechselspiels des Gelenkes und der Muskulatur sowie zur Erhöhung des intraartikulären Druckes [18,19 ]. Bei einer bundesweiten Umfrage 1994 war bei 20\% der befragten Operateure die Watson-Jones-Plastik das Verfahren der ersten Wahl (20). In Deutschland sind mehr als 10 Modifikationen bekannt. Wir bevorzugen die Watson-Jones-Plastik in der Modifikation nach Holz und Weller [25].

\section{Op-Technik}

Lateraler Zugang, distal nach vorne umbiegend. Ein ca. $13 \mathrm{~cm}$ langer Span aus der Peroneus brevis-Sehne wird proximal durchtrennt und mit einem Haltefaden armiert. Der Span umfasst etwa ein Drittel des Sehnendurchmessers und bleibt distal gestielt. Es werden zwei parallele 4,5-mm-Bohrkanäle in der Außenknöchelspitze und ein weiteres V-förmiges Bohrloch am lateralen Talushals an der Knorpel-/Knochengrenze angelegt. Nach Durchziehen der Sehne mit Fädeldrähten

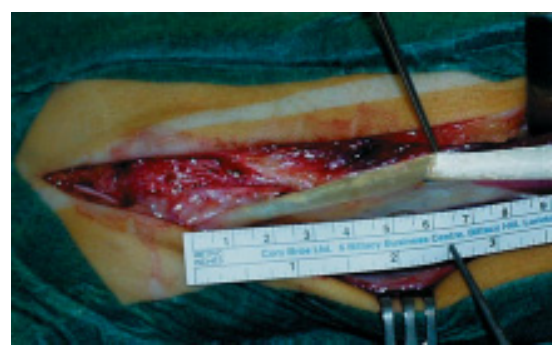

Abb.13 Präparation eines $13 \mathrm{~cm}$ langen Spans der peroneus brevis Sehne. 


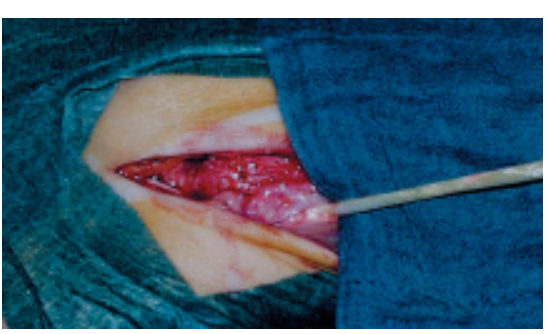

Abb.14 distal gestielter, proximal durchtrennter Sehnenspan.

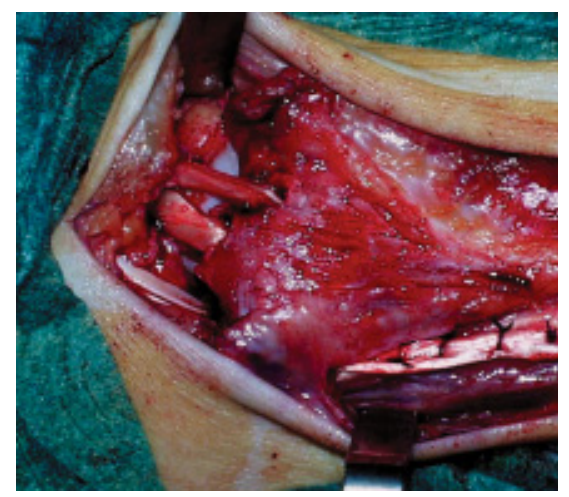

Abb.17 Ohne Spannung mit sich vernähter Sehne.

wird diese bei physiologischer Sprunggelenksstellung ohne Spannung mit sich selbst mit resorbierbarem Nahtmaterial (3/0) vernäht.

Postoperativ wird das Sprunggelenk im zirkulären Unterschenkelliegegips ruhiggestellt, am 2.postoperativen Tag ein Wund- und Bewegungsfenster angelegt. Dadurch sind die Extension und Flexion im oberen Sprunggelenk frei gegeben. Nach Entfernung des Nahtmaterials am 11.Tag wird ein Unterschenkelgehgips bis zum Ablauf der 4. postoperativen Woche angelegt.

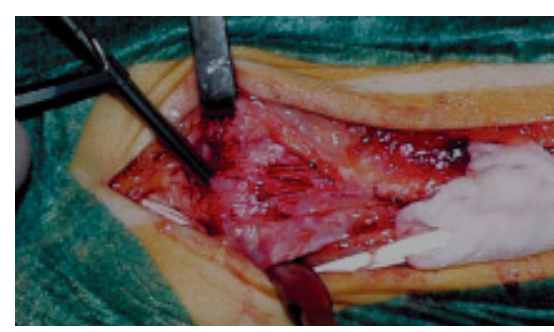

Abb.15 Anlegen der 4,5-mm-Bohrlöcher an der Fibulaspitze.

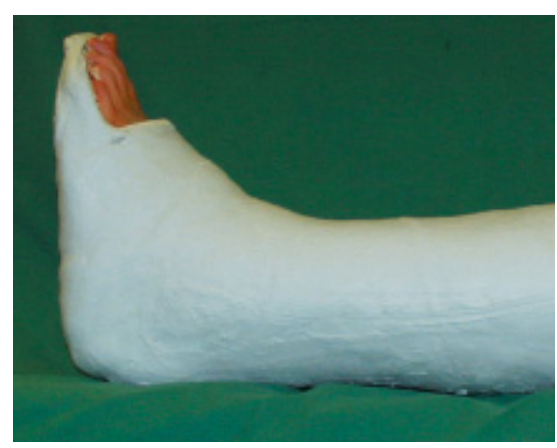

Abb.18 Postoperative Ruhigstellung im zirkulären US-Liegegips.

Für die Watson-Jones-Plastik sind gute Ergebnisse aus der Literatur bekannt, auch die jüngsten Veröffentlichungen bescheinigen diesem Verfahren exzellente Ergebnisse (21,13). Aus dem eigenen Patientengut konnten 21 von 29 Patienten bei einem durchschnittlichen Follow-up von 21 Monaten auf der Grundlage des Zwipp-Scores (objektiv, subjektiv, sportphysiologisch und radiologisch) nachuntersucht werden. Bei 12 Patienten fand sich ein sehr gutes, bei 7 Patienten ein gutes und bei 2 ein mäßiges Resultat (Abb.11-19).

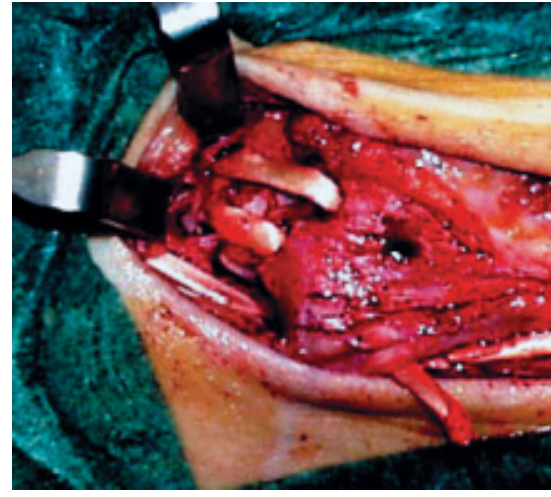

Abb.16 Durchzug der Sehne durch die Bohrlöcher.

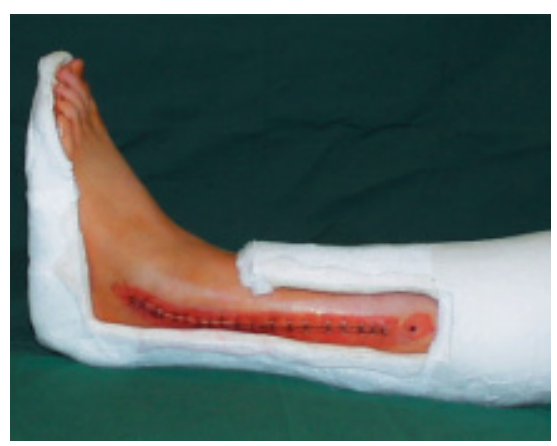

Abb.19 US-Liegegips mit Wund- und Bewegungsfenster.

\section{Konservative Therapie}

Die funktionelle Instabilität des OSG wird konservativ behandelt: Orthesen haben kurzfristig einen positiven Effekt. Nachgewiesen sind die Besserung der Standund Gangunsicherheit und ein positiver Effekt auf die Propriozeption. Die Erhöhung des lateralen Schuhrandes um ca. $7 \mathrm{~mm}$ korrigiert das Gangbild. Gangschule, Koordinationstraining und Reflextraining für die Pronatoren sind das allgemein akzeptierte Therapie-Schema $[22,23,24]$. 


\section{Literatur}

${ }^{1}$ Soames RW. Skeletal system. In: Williams PL, Bannister LH, Berry MM,Collins P, Dyson M, Dussek JE, Ferguson MWJ (eds). Gray's anatomy. The anatomical basis of medicine and surgery, 38th ed. Churchill Livingstone, New York, 1995; 425-736

2 Debrunner HU, Jacob AC. Biomechanik des Fußes. Ferdinand Enke Verlag Stuttgart 1998

${ }^{3}$ Dehne E. Die Klinik der frischen und habituellen Adduktions-supinations-distorsion des Fußes. Dtsch Z Chir 1933; 242: 40-61

${ }^{4}$ Bozic R, Weiser J. Epidemiologic data of rupture of the fibular ligament of the upper ankle joint. Aktuelle Traumatol 1991; 21 (3): $118-120$

${ }^{5}$ Holmer P, Sondergaard L, Koradsen L, Nielsen PT, Jorgensen LN. Epidemiology of Sprains in the lateral ankle and foot. Foot Ankle Int 1994; 15 (2): $72-74$

${ }^{6}$ Lynch SA, Renstrom PA. Treatment of acute lateral ankle ligament rupture in the athlete. Conservative versus surgical treatment. Sports Med 1999; 27 (1): $61-71$

${ }^{7}$ Marder R. Current methods for the evaluation of ankle ligament injuries. J Bone Joint Surg 1994; 76A: $1103-1111$

${ }^{8}$ Breitenseher MJ, Trattnig S, Kukla C, Gäbler C, Kaider A, Baldt M, Haller J, Imhof H. MRI versus lateral stress radiography in acute lateral ankle ligament injuries. J Comp Assist Tomogr 1997; 21: 280-285

${ }^{9}$ Zwipp H. Chirurgie des Fußes. Springer-Verlag Wien NewYork 1994

${ }^{10}$ Wülker N, Rudert M. Fibulare Kapsel-BandRupturen. Der Orthopäde 1999; 28: $476-$ 482

${ }^{11}$ Kelikian H, Kelikian AS. Disorders of the ankle. Saunders, Philadelphia 1985; 339-363
${ }^{12}$ Zwipp H et al. Zur radiologischen Diagnostik der anterolateralen Rotationsinstabilität im OSG. Unfallheilkunde 1982; 85: 419-426

${ }^{13}$ Von Pijnenburg ACM, Van Dijk CN, Bossuyt PMM. Treatment of Ruptures of the Lateral Ankle Ligaments: A Meta-Analysis. J Bone Joint Surg Br 2000; 82 (6): 761 - 773

${ }^{14}$ Löfvenberg R, Karrholm J, Lund B. The outcome of nonoperated patients with chronic lateral instability of the ankle. A 20-years follow-up study. Foot Ankle 1994; 15: $165-169$

${ }^{15}$ Freeman MAR, Dean MRE, Hanham IWF. The etiology and prevention of functional instability of the foot. J Bone Joint Surg Br 1965; 47: $678-685$

${ }^{16}$ Michelson JD, Hutchins C. Mechanoreceptors in human ankle ligaments. J Bone Joint Surg Br 1995; 77: 219-224

${ }^{17}$ Becker HP, Rosenbaum D, Claes L, Gerngroß $H$. Dynamische Pedographie zur Abklärung der funktionellen Sprunggelenksinstabilität. Unfallchirurg 1997; 100: 133-139

${ }^{18}$ Rosenbaum D, Becker HP, Wilke HJ, Claes L. Tenodeses destroy the kinematic coupling of the ankle joint complex. A three-dimensional in vitro joint motion analysis. J Bone Joint Surg Br 1998; 80: 162 -68

${ }^{19}$ Rosenbaum D, Bertsch C, Claes L. Tenodeses do not fully restore ankle joint loading characteristics: A biomechanical in vitro investigation of the hind foot. Clin Biomech 1997; 12: $202-209$

${ }^{20}$ Becker HP, Schmidt R, Gutcke A, Gerngroß H. Aktueller Stand der Diagnostik und Therapie der chronischen Außenbandinsuffizienz am Sprunggelenk - Ergebnisse einer Umfrage an 267 Kliniken in Deutschland 1994. Unfallchirurg 1995; 98: $493-498$

${ }^{21}$ Sugimoto K, Takakura Y, Akiyama K, Kamei S, Kitada C, Kumai T. Long-term results of Wat-
son-Jones tenodesis of the ankle. J Bone Joint Surg Am 1998; 80: 1587-1596

${ }^{22}$ Bernier JN, Perrin DH. Effect of coordination training on proprioception of the functionally unstable ankle. J Orthop Sports Phys Ther 1998; 27: 264-275

${ }^{23}$ Jerosch N, Bischof M. The effect of proprioception on functional stability of the upper ankle joint with special reference to stabilizing aids. Sportverletz Sportschaden 1994; 8: $111-121$

24 Pintsaar A. Postural corrections after standardised perturbations of single limb stance: effect of training and orthotic devices in patients with ankle instability. $\mathrm{Br} \mathrm{J}$ Sports Med 1996; 30: 259-263

${ }^{25}$ Holz U. Die chronische Außenbandinstabilität am oberen Sprunggelenk. Operat. Orthop. Traumatol. 1989; 1: 69-74

\section{Dr. med. Dara Orangi \\ Assistenzarzt \\ Prof. Dr. med. Karl Heinrich Winker Chefarzt}

Klinik für Unfall-, Hand-

und Wiederherstellungschirurgie

Klinikum Erfurt

Nordhäuser Str. 74

99089 Erfurt 